\title{
Validation of a social deprivation index and association with cognitive function and decline in older adults
}

\author{
Lena M. Hofbauer (1) and Francisca S. Rodriguez \\ German Center for Neurodegenerative Diseases (DZNE), Ellernholzstr 1-2, 17489 Greifswald, Germany
}

ABSTRACT

Objectives: Previous work using a US sample has shown that an index of social deprivation (SoDep Index) is associated with cognitive functioning and decline in older adults. This study aimed to replicate these findings using a European sample (Survey of Health, Ageing and Retirement in Europe, SHARE).

Design: We analyzed data of 51,630 respondents aged 50 years and older (M: 63.5 years, standard deviation [SD]: 9.1) with at least two cognitive assessments (follow-up M: 6.06 years, SD: 3.86). Cognitive scores were transformed to Z-scores. Multiple growth curve modeling was used to model cognitive status and decline as predicted by the SoDep Index. In a sensitivity analysis, we constructed a new SoDep Index (SoDep Index new $_{\text {) }}$ including further social deprivation domains.

Results: Adjusting for covariates, a unit increase in SoDep Index was associated with a cognitive score of 0.037 SDs smaller $(p<.001)$ and a decline 0.003 SDs per year faster $(p<.001)$. Of the covariates, depressive symptoms, chronic disease burden, male gender, and widowhood were also associated with poorer cognition. Being divorced was associated with better cognition. Sensitivity analysis confirmed findings. Compared to the SoDep Index, the SoDep Index ${ }_{\text {new }}$ showed a more pronounced association with both cognition and cognitive decline.

Conclusions: We were able to replicate results showing an association between SoDep Index and cognitive function and decline. The sensitivity analysis further emphasizes the relevance of financial security. This strengthens the implication that preventing social deprivation can contribute to reducing the dementia burden by raising cognitive functioning in the older population. The findings are relevant to policy-makers and health care practitioners.

Keywords: cognitive impairment, dementia, epidemiology, longitudinal studies

\section{Introduction}

Social deprivation has been defined as a deprivation in socioeconomic resources relative to what is considered standard in a given society (Townsend et al., 1988). While socioeconomic status is rather narrowly operationalized in terms of education, occupation, and income (Galobardes et al., 2006), the social deprivation construct includes any socioeconomic domain relevant to a given societal context, for example, adequate housing or food security (Townsend et al., 1988). Central to the definition is that social deprivation excludes individuals from society. To combat this, the European Commission

Correspondence should be addressed to: Lena M. Hofbauer, German Center for Neurodegenerative Diseases (DZNE), Research Group 'Psychosocial Epidemiology and Public Health', Ellernholzstr. 1-2, 17489 Greifswald, Germany, Phone: + 4903834867603; Fax: +49 38348619 551; Email: lena.hofbauer@dzne.de. Received 26 Mar 2021; revision requested 13 May 2021; revised version received 14 Jun 2021; accepted 06 Jul 2021. First published online 08 September 2021 aimed to reduce the number of socioeconomically excluded people in the Union by 20 million in the decade from 2010 to 2020 (European Commission, 2010). By 2018, data suggested that this goal had not yet been met and members of the Commission noted that the COVID-19 pandemic spreading across Europe in 2020 made this increasingly unlikely (Becker et al., 2020).

Diminishing social deprivation would unburden health care systems, as the relationship between indicators of social deprivation and a range of health outcomes, such as cardiovascular disease and cancer, is well established (Mackenbach et al., 2008). Furthermore, reducing social deprivation would also help reduce the European dementia burden. Social deprivation has the potential to lower dementia incidence via two pathways. On the one hand, a reduction in social deprivation can be expected to reduce the cumulative deprivation-associated health risk. Given a lower overall disease burden, dementia 
risk would be reduced (Ward et al., 2021). On the other hand, reducing social deprivation could increase brain maintenance. The experience of chronic stressors is considered particularly damaging (Lupien et al., 2005). Reducing social deprivation should reduce the chronic stressors experienced by individuals and thus improve cognitive reserve.

Studies have shown an association between markers of socioeconomic status and cognitive functioning (Chiao et al., 2014; Lövdén et al., 2020; Lyu and Burr, 2016; Marden et al., 2017) and cognitive decline (Lyu and Burr, 2016; Marden et al., 2017). However, the use of standard socioeconomic status indicators with older populations has been challenged (Grundy and Holt, 2001; O'Reilly, 2002). On the one hand, indicators relevant to younger populations may show less of an association to health outcomes in older age. For instance, income becomes less relevant toward retirement, when accumulated wealth is increasingly relied on (Galobardes et al., 2006). On the other hand, standard measures only assess a subset of deprivation domains. As such, they do not capture the accumulative effect of overlapping deprivation in multiple domains (Bradshaw and Finch, 2003). Multidimensional social deprivation measures have, therefore, recently gained attention in predicting health outcomes in older adults (Chung et al., 2018; Pförtner and Elgar, 2016; Saito et al., 2014).

Yet, these investigations do not include cognitive outcomes. Thus, there is a dearth of direct evidence of an association between social deprivation and cognition. A survey of French older adults ( $>60$ years old) addressed this by assessing social deprivation by a questionnaire on a greater range of deprivation domains (e.g. lack of supplementary health insurance coverage, lack of social support, not having been on a holiday). The findings showed an association between social deprivation and self-reported cognitive decline (Bongue et al., 2016). In an effort to establish a possible association between social deprivation as a multidimensional construct and cognitive decline, we recently analyzed data of a sample of over 11,000 respondents over the age of 50 years in the longitudinal, nationally representative US Health and Retirement Study (HRS) using objective cognitive outcome measures. We constructed a social deprivation index (SoDep Index) covering the domains' education, income, wealth, health insurance status, job stability, and neighborhood safety. We found that this SoDep Index predicted cognitive function and, to a lesser degree, cognitive decline (Hofbauer and Rodriguez, 2021), indicating that preventing social deprivation might indeed contribute to raising cognitive functioning in the older population.

Given that the resources most relevant to deprivation will vary across societies, these findings based on a US sample may not generalize to other populations. For instance, the US health care system is more heterogeneous than European health care systems (Garber and Skinner, 2008), possibly creating a greater relevance of health insurance status to health outcomes in the US than would be seen in Europe. Conversely, the relevance of job stability may be higher in Europe than in the US. The US job market is characterized by greater dynamism, leading to an overall higher job mobility than in Europe. Therefore, short job tenures are more common and temporary job loss is less likely to result in stable unemployment (Storm and Naastepad, 2009).

In this paper, we therefore aimed to validate the SoDep Index that we derived from the US American sample in a European sample, the Survey of Health, Ageing and Retirement in Europe (SHARE). We replicated the SoDep Index for Europe and expected the findings to confirm that greater scores on this SoDep Index are indeed associated with poorer cognitive function and faster cognitive decline. In addition, we conducted a sensitivity analysis, in which we constructed a new, more comprehensive SoDep Index (SoDep Index new $_{\text {), }}$ based on additional social deprivation domains available in the SHARE, and repeated the main analyses.

\section{Methods}

\section{Sample}

The SHARE cross-country study is based on a sample of Europeans (and Israelis) over the age of 50 years and their partners. It started in 2004 (wave 1 ), and interviews have been taking place approximately every 2 years. At the time of writing, the most recent data available were collected in 2017 (wave 7). The Ethics Committee of the University of Mannheim approved SHARE waves 1-4. The Ethics Council of the Max Planck Society approved waves 4-7. All participants provided written informed consent. For each respondent, baseline data on social deprivation domains and covariates from the year of their initial cognitive assessment were used. As no cognitive testing was completed in the third wave, this includes all waves except wave 3. In addition, we extracted employment history data from the retrospective life course (SHARELIFE) interviews conducted in the third and the seventh wave. We included respondents for whom social deprivation and covariate information were available and who, in the year of their first cognitive testing, were at least 50 years old. We further excluded individuals who, at the baseline assessment, were institutionalized. Finally, respondents were only 


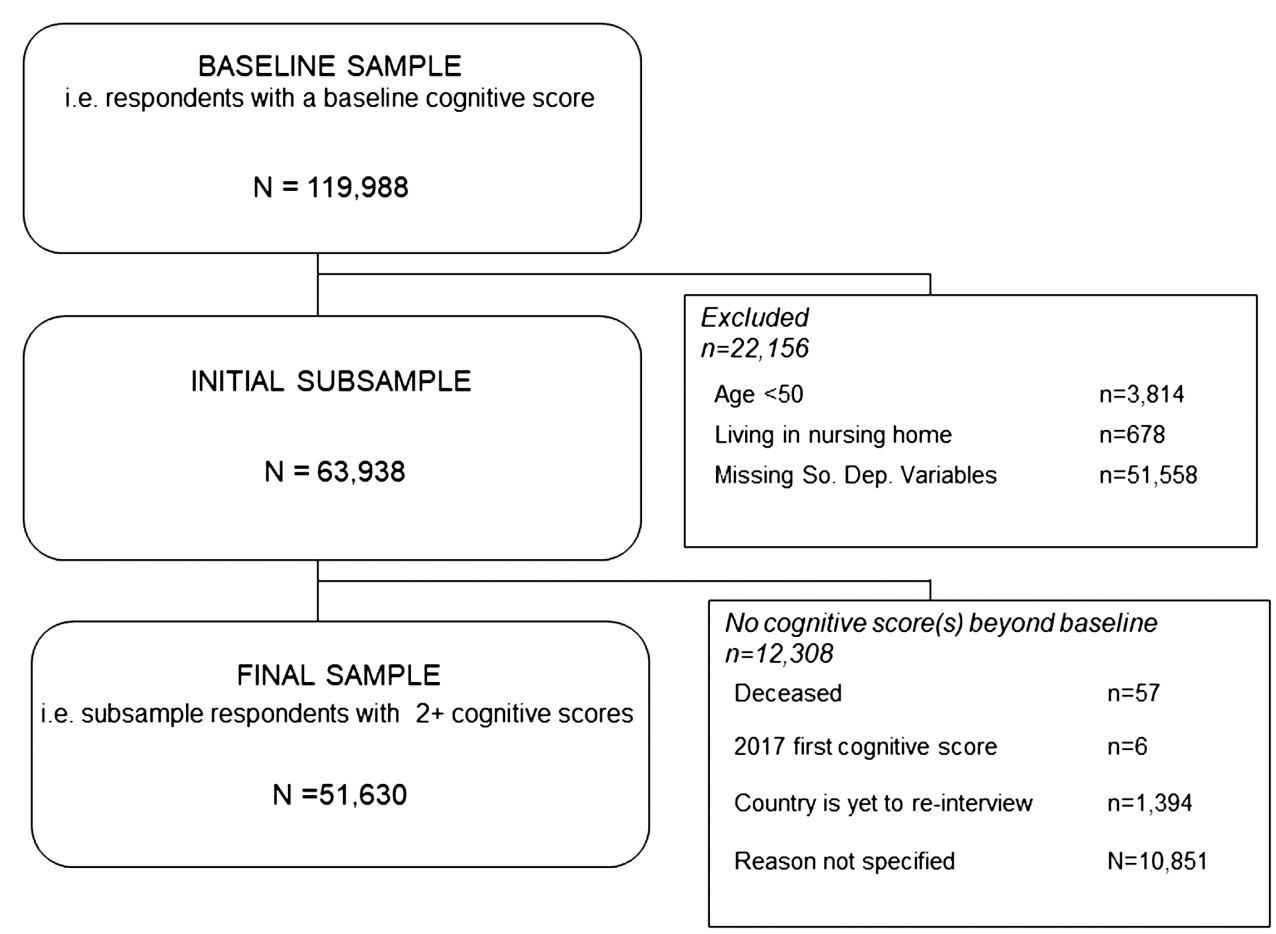

Figure 1. Flow chart representing the selection of the final analysis sample. After exclusion of those under 50 years of age, living in nursing homes, or for whom social deprivation information was missing, we further excluded those without cognitive follow-up. The final sample size is $N=51,630$. Abbreviations: So. Dep., social deprivation.

included if they had at least two cognitive test scores. The final sample contained 51,630 respondents (see Figure 1).

\section{Social deprivation index (SoDep Index)}

We calculated SoDep Index scores by summing the previously established domains weighted by standardized structural equation modeling (SEM) coefficients for each (see Table $S 1$ published as supplementary material online attached to the electronic version of this paper). The SoDep Index was constructed using data from the US HRS, as reported previously (Hofbauer and Rodriguez, 2021). It contains information on education, job stability, income, wealth, health insurance status, and neighborhood safety. Due to lacking information in the SHARE data, we had to omit neighborhood safety as a SoDep Index domain. The included domains are the following:

\section{YEARS OF EDUCATION}

The years of education completed (range: 0-25) reverse-coded so that a higher score indicates higher deprivation.

\section{Job STABILITY}

We used the SHARELIFE job episodes panel data, which gives information on respondents' employment year by year to determine whether or not a respondent reported ever having held a job for 5 or more years (coding Yes $=1, \mathrm{No}=2$ ).

\section{INCOME}

We used the SHARE-provided generated value of total household income (thinc), which sums all reported household incomes. We adjusted this value for the household size by dividing it by the square root of household size. This is a common adjustment accounting for the nonlinear growth in financial needs with growing household size (Chanfreau and Burchardt, 2008). The values were transformed using the inverse hyperbolic sinus (IHS) transformation. Values were then reverse-scored for the purpose of generating the SoDep Index, so that greater values indicate increased deprivation.

WEALTH

SHARE provides a generated variable (hnfass) that expresses a household's net financial assets less the household's financial liabilities. We adjusted for household size by dividing it by the square root of the number of household members, IHStransformed the values, and reverse-scored them.

Health insurance status

We recorded whether a respondent reported having any supplementary health insurance with increased 
access to health care services (coding Yes $=1$, $\mathrm{No}=2$ ), as opposed to only having general public health insurance. Since respondents were only asked about supplementary health insurance in waves 1,5 , 6 , and 7 , we transferred the answer from wave 1 to waves 2 and 4 (no data from wave 3 is used).

\section{SoDep Index $x_{\text {new }}$ variables for the sensitivity analysis}

The SoDep Index domains (see Table S1) used in the main analysis were limited by the availability of relevant domains in the HRS data set used for the original index construction. They are not considered to be exhaustive. For this reason, we conducted a sensitivity analysis. In this analysis, we added further candidate domains in a SEM to construct a more comprehensive index (SoDep Index new $_{\text {w }}$ ). As additional candidates, we considered 27 items that were designed by SHARE specifically to describe deprivation (Stuck et al., 2020). Some of these items could not be used because the majority of respondents had missing data (e.g. questions regarding ability to afford fruit/vegetables, weekly social activities). The 24 items remaining were included in the SEM modeling of the sensitivity analysis (details in File S1).

\section{Covariates}

Covariates recorded in the year of the respondents first available cognitive function score were gender, the number of chronic health conditions respondents had ever been diagnosed with, their marital status, and the harmonized depression scale (EURO-D) score (for details see Table 1). It has been established that being married or partnered has beneficial effects on cognition (Sommerlad et al., 2018). Depressive symptoms, on the other hand, are a well-established risk factor in cognitive decline (Cherbuin et al., 2015). Evidence on gender effects in cognitive aging is inconsistent. While early evidence showed a higher incidence of dementia in women (Gao et al., 1998), a recent meta-analysis found no difference in incidence or prevalence (Fiest et al., 2016). Others report superior cognitive function in older women compared to men (Bloomberg et al., 2021). This may be partially due to women in more recently born cohorts having had greater educational and occupational opportunities, reducing socioeconomic disparity and increasing their ability to build and/or maintain cognitive reserve (Nebel et al., 2018).

\section{Outcome measure}

Cognitive testing in the SHARE assessed word recall, orientation, numeracy, and verbal fluency.
Table 1. Sample demographics. Descriptive statistics of baseline covariates, cognitive testing results, and social deprivation variables

\begin{tabular}{|c|c|}
\hline Total $\mathrm{N}=$ & 51,630 \\
\hline \multicolumn{2}{|l|}{ Age } \\
\hline Mean (SD) & $63.5(9.1)$ \\
\hline Range & $50-101$ \\
\hline \multicolumn{2}{|l|}{ Baseline covariates } \\
\hline \multicolumn{2}{|l|}{ Gender } \\
\hline Male: Female & 24,130: $27,500(46.74 \%$ male $)$ \\
\hline \multicolumn{2}{|l|}{ Marriage status } \\
\hline Married/partnered & $38,474(75.52 \%)$ \\
\hline Widowed & $5,808(11.25 \%)$ \\
\hline Divorced & $4,469(8.66 \%)$ \\
\hline Never married & $2,879(5.58 \%)$ \\
\hline \multicolumn{2}{|l|}{ Chronic conditions ( $n$ ) } \\
\hline Mean (SD) & $1.65(1.51)$ \\
\hline Range & $0-13$ \\
\hline \multicolumn{2}{|l|}{ EURO-D score } \\
\hline Mean (SD) & $2.31(2.16)$ \\
\hline Range & $0-12$ \\
\hline \multicolumn{2}{|l|}{ Cognitive follow-up (years) } \\
\hline Mean (SD) & $6.06(3.86)$ \\
\hline Range & $1-13$ \\
\hline \multicolumn{2}{|l|}{ SoDep Index variables } \\
\hline \multicolumn{2}{|l|}{ Education (years) } \\
\hline Mean (SD) & $10.91(4.37)$ \\
\hline \multicolumn{2}{|l|}{ Income $^{a}$} \\
\hline Median (IQR) & $23,335(36,681)$ \\
\hline Range & $0-5,477,950$ \\
\hline \multicolumn{2}{|l|}{ Wealth $^{a}$} \\
\hline Median (IQR) & $6,615(41,811)$ \\
\hline Range & $-4,225,787$ to $9,510,352$ \\
\hline \multicolumn{2}{|l|}{ Health insurance } \\
\hline National insurance only & $45,273(87.69 \%)$ \\
\hline Supplementary insurance & $6,357(12.31 \%)$ \\
\hline \multicolumn{2}{|l|}{ Ever held a job for $5+$ years } \\
\hline Yes & $50,060(96.96 \%)$ \\
\hline No & $1,570(3.04 \%)$ \\
\hline
\end{tabular}

Note. Descriptive statistics are presented as means with standard deviations (SD) or medians with interquartile ranges (IQR), as appropriate. Ranges (minimum-maximum) or percentages of the final sample $(\mathrm{N}=51,630)$ are also presented.

${ }^{a}$ Values in Euros, rounded.

The format of the word recall was adapted from the modified Telephone Interview of Cognitive Status (Brandt et al., 1988). Respondents were asked to reproduce a list of 10 words, both immediately and after a delay (approx. 5 minutes). Each correctly recalled word was worth one point at each instance (range: 0-20). For a measure of their orientation, respondents were asked about the current date (scored 0-4, one point each for correct day of the month, month, year, and day of the week). Numeracy was assessed using four questions on percentages (scored 0-4, one point for each correct answer). For the verbal fluency test, respondents 
list the names of as many animals as they can think of in 1 minute (range: 0-100). Across all waves of testing, we converted the raw scores for each domain to $\mathrm{Z}$-scores and averaged them to yield a global cognitive Z-score - a common approach to derive a measure on cognitive functioning, see for example Bennett et al. (2006).

\section{Statistical analyses}

Descriptive ANALyses

We calculated the mean and standard deviation (SD) or the median (interquartile range) of participant demographics. Further, we calculated the countrywise percentages of individuals reporting deprivation on the SoDep Index domains. For the continuous variables (income, wealth, and education), we describe the lowest $20 \%$ as deprived.

Modeling of Cognitive status ANd DeCline We conducted growth curve modeling using the nlme package in $\mathrm{R}$. All models include a random intercept and a random slope allowing for the initial cognitive status and the association between time and cognitive score to vary randomly. Age was used as the time variable and re-centered at 50 . We used the modeled coefficients of an unconditional means model (Model 1) to calculate the intraclass correlation coefficient (ICC). An ICC $>0.25$ indicates high between-person variability, and thus implies that multilevel modeling is indeed appropriate. The unconditional growth model (Model 2) was fit to assess whether there is sufficient variation in cognition across ages. A model containing a quadratic age term (Model 3) was employed to assess whether the relationship can indeed be assumed to be linear. Model fit was judged by Akaike information criteria (AIC) and the Bayesian information criteria (BIC). Since we saw only a marginal improvement, we decided on the most parsimonious approach: we included the squared age term but only investigated the linear interaction with SoDep Index in an unadjusted conditional model (Model 4). We then added in all covariates into the second conditional model (Model 5) to determine whether any association between SoDep Index and cognitive scores would survive adjustment for these.

\section{EFFECT ESTIMATION}

We used the parameters of the adjusted conditional model to calculate the predicted cognitive scores at ages 60 and 80 years for individuals with a score marking the bottom or top $20 \%$ of SoDep Index values, respectively. In this, we kept categorical variables at the reference level (gender: female; marriage status: married/partnered) and used the average values for the number of chronic conditions and EURO-D score (see Table 1).

\section{SENSITIVITY ANALYSIS}

The majority of SHARE deprivation domains (see File S1) were only introduced in wave 5 . We thus used a subset of participants who completed cognitive baseline measures in wave $5(n=23,099)$ in our sensitivity analysis. Among these, we excluded individuals younger than 50 years of age $(n=599)$ and those who were institutionalized $(n=215)$. Moreover, respondents with missing SHARE deprivation variables were excluded $(n=15,710)$. Finally, we excluded those who did not have a follow-up cognitive assessment in the following waves $(\mathrm{n}=497)$. As a result, 6,078 respondents were included in the sensitivity analysis.

SEM was conducted using lavaan in $\mathrm{R}$ to build the new SoDep Index (SoDep Index $\left.{ }_{\text {new }}\right)$. To assess the fit, we considered three separate fit measures: the comparative fit index (CFI), the root mean square error of approximation (RMSEA), and the standardized root mean square residual (SRMR). Thresholds commonly used to determine acceptable fit are CFI $>0.95$, RMSEA $<0.06$, and SRMR $<0.8$ (Hooper et al., 2007). We initially built a model that included all of the social deprivation dimensions loading onto a single social deprivation construct (SEM1, see Table S2). However, the fit was poor. Inspecting the residual correlations revealed two major sources of fit issues. First, the model consistently and notably overestimated the correlation between some candidate variables with the other candidates (residual correlations $<-0.2$ with five or more other candidates). This suggests that the variables are not, after all, good candidates. They were removed (see Table S2). In addition, the model notably underestimated the correlation between some candidate variables. We inspected modification indices to see which correlations, if added, would make a notable change to the overall model fit (Mueller and Hancock, 2008). Correlations which made theoretical sense were added: between (1) no helpful people in the local area and not feeling part of the local area, (2) vandalism/crime in the local area and the local area being not clean, (3) low income and low health insurance status, (4) low income and inability to afford a holiday, and (5) the inability to replace worn-out clothes and the inability to replace worn-out shoes. The resulting second model (SEM2) had a reasonably good fit, yet some variables had loadings $<.4$ onto the social deprivation construct (see Table S2). Removing these variables resulted in further improved fit $(\mathrm{CFI}=1.00$, $\mathrm{RMSEA}=0.02$, and SRMR $=0.03)$ and significant loadings $>.4$ in all 
Table 2. Countrywise percentages of respondents reporting relative deprivation in the SoDep Index domains

\begin{tabular}{|c|c|c|c|c|c|c|}
\hline COUNTRY & $\mathrm{N}$ & $\begin{array}{c}\% \text { Low } \\
\text { EDUCATION }\end{array}$ & $\begin{array}{l}\% \text { Low } \\
\text { INCOME }\end{array}$ & $\begin{array}{l}\% \text { Low } \\
\text { WEALTH }\end{array}$ & $\begin{array}{c}\% \text { NO SUPPLEMENTARY } \\
\text { HEALTH INSURANCE }\end{array}$ & $\begin{array}{c}\% \text { Never HELd a JOB FOR } \\
5+\text { YeARS }\end{array}$ \\
\hline Austria & 3,259 & 24.21 & 7.43 & 14.73 & 91.72 & 4.11 \\
\hline Belgium & 4,587 & 14.21 & 6.19 & 11.66 & 47.61 & 4.53 \\
\hline $\begin{array}{l}\text { Czech Re- } \\
\text { public }\end{array}$ & 4,226 & 2.96 & 53.17 & 22.5 & 99.24 & 0.4 \\
\hline Denmark & 3,395 & 11.1 & 1.41 & 14.29 & 70.87 & 3.3 \\
\hline Estonia & 4,236 & 12.13 & 67.23 & 20.8 & 99.95 & 0.54 \\
\hline France & 3,692 & 20.69 & 6.18 & 15.17 & 61 & 4.2 \\
\hline Germany & 4,248 & 3.51 & 6.31 & 12.48 & 75.28 & 3.58 \\
\hline Greece & 1,831 & 42.55 & 31.68 & 44.57 & 94.76 & 1.47 \\
\hline Israel & 1,427 & 18.57 & 19.76 & 38.33 & 53.96 & 2.24 \\
\hline Italy & 3,377 & 41.78 & 14.42 & 27.27 & 95.62 & 2.87 \\
\hline Luxembourg & 723 & 10.24 & 2.35 & 12.72 & 21.85 & 5.26 \\
\hline $\begin{array}{l}\text { The Nether- } \\
\text { lands }\end{array}$ & 1,880 & 10.69 & 4.2 & 11.28 & 47.23 & 7.23 \\
\hline Poland & 1,556 & 35.09 & 85.67 & 55.85 & 100 & 1.99 \\
\hline Portugal & 882 & 72 & 49.55 & 22.79 & 100 & 1.59 \\
\hline Slovenia & 1,976 & 11.08 & 26.27 & 34.06 & 77.63 & 2.18 \\
\hline Spain & 4,064 & 45.99 & 25.76 & 23.01 & 91.31 & 2.83 \\
\hline Sweden & 3,665 & 21.8 & 1.99 & 17.6 & 86.52 & 1.06 \\
\hline Switzerland & 2,606 & 40.02 & 5.33 & 8.9 & 89.72 & 7.56 \\
\hline
\end{tabular}

Note. The number of respondents included from each country are shown. Based on these, percentages of respondents meeting deprivation criteria were calculated for each country. To calculate the percentage of low education respondents, all respondents who received 7 years or less of education (i.e. the cross-country $20 \%$ quantile) were identified. Low-income respondents were those that had an income of up to $8,468.674$ Euros (i.e. cross-country $20 \%$ quantile) and those who had income of up to 0.00001 Euros of wealth (i.e. cross-country $20 \%$ quantile) were classed as low wealth.

cases. Domains included in this final SEM were income, wealth, inability to afford groceries, inability to afford a holiday, inability to afford an unexpected expense, inability to replace worn-out clothing, inability to replace worn-out shoes, and missing a necessary doctor appointment to save costs (see Table S2). The latent SoDep Index new $_{\text {ne }}$ score for each respondent was predicted using this final SEM and appended to the data set. The main analyses were repeated using SoDep Index $x_{\text {new }}$.

\section{Results}

\section{Descriptive analyses}

At baseline, respondents had an average age of 63.5 years (SD: 9.1). The cognitive follow-up lasted on average 6.06 (SD: 3.86) years. For more descriptive statistics, see Table 1 . There exists great variation in terms of the deprivation domains between countries (see Table 2). A relatively high rate of low education is notable in Greece, Italy, Poland, Spain, and Switzerland. Income deprivation is most common in the Czech Republic, Greece, Estonia, Poland, and Portugal. Greece, Israel, Poland, and Slovenia show the most notable rates of low wealth in their populations. The rate of individuals who did not possess supplementary health insurance was highest in the Czech Republic, Estonia, Italy, Poland, and Portugal. There was only minimal variation in the percentage of respondents who never held a job for $5+$ years, speaking to generally high levels of 5 -year job stability across Europe.

\section{Cognitive functioning and decline}

Table 3 presents the results of growth curve models investigating the association between the social deprivation index (SoDep Index) and cognitive scores. Model 1 presents the unconditional means model, and it shows that there is significant variation in cognitive scores $(\mathrm{ICC}=0.64)$. The unconditional growth model (Model 2) showed that cognitive scores changed significantly over time. Model 3 showed a significant quadratic association between age and cognitive score and AIC and BIC indicate an improved fit. The conditional model with the SoDep Index as a predictor (Model 4) shows that the greater SoDep Index values were associated with poorer cognition as well as a faster cognitive decline. 
Table 3. Results from linear growth models of the associations between SoDep Index and cognitive scores

\begin{tabular}{|c|c|c|c|c|c|c|c|}
\hline & M1 & M2 & M3 & M4 & & M5 & \\
\hline Intercept & $0.08(0.00)^{* * *}$ & $0.35(0.00)^{* * *}$ & $0.20(0.00)^{* * * *}$ & $0.89(0.01)^{* * *}$ & & $0.99(0.01)^{* * *}$ & \\
\hline Age & & $-0.02(0.00)^{* * *}$ & $0.01(0.00)^{* * *}$ & $0.02(0.00)^{* * *}$ & & $0.02(0.00)^{* * * *}$ & \\
\hline $\mathbf{A g e}^{2}$ & & & $-0.00(0.00)^{* * *}$ & $-0.00(0.00)^{* * * *}$ & & $-0.00(0.00)^{* * *}$ & \\
\hline & & & & Score & Cog. Dec. & Cog. Score & Cog. Dec. \\
\hline SoDep Index & & & & $-0.04(0.00)^{* * * *}$ & $-0.00(0.00)^{* * *}$ & $-0.03(0.00)^{* * * *}$ & $-0.00(0.00)^{* * *}$ \\
\hline Gender: male ${ }^{a}$ & & & & & & $-0.11(0.00)^{* * *}$ & \\
\hline Marriage status: widowed $^{\mathrm{b}}$ & & & & & & $-0.08(0.01)^{* * *}$ & \\
\hline Marriage status: divorced/separated ${ }^{\mathrm{b}}$ & & & & & & $0.12(0.01)^{* * *}$ & \\
\hline Marriage status: never married ${ }^{\mathrm{b}}$ & & & & & & $-0.01(0.00)$ & \\
\hline No. of chronic conditions & & & & & & $-0.02(0.00)^{* * *}$ & \\
\hline EURO-D score & & & & & & $-0.04(0.00)^{* * *}$ & \\
\hline \multicolumn{8}{|l|}{ Goodness of fit } \\
\hline AIC & 203119 & 194462 & 192321 & 182232 & & 179625 & \\
\hline BIC & 203159 & 194532 & 192431 & 182332 & & 179785 & \\
\hline
\end{tabular}

Abbreviations: Cog. = cognitive; Dec $=$ decline

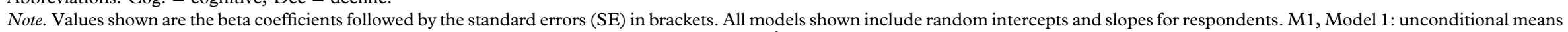

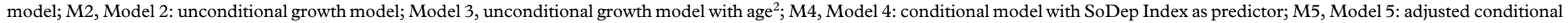
model with covariates.

${ }^{a}$ Gender: female is the reference level.

${ }^{\mathrm{b}}$ Marriage status: married/partnered is the reference level.

${ }^{* * *} \mathrm{p}<.001$ 


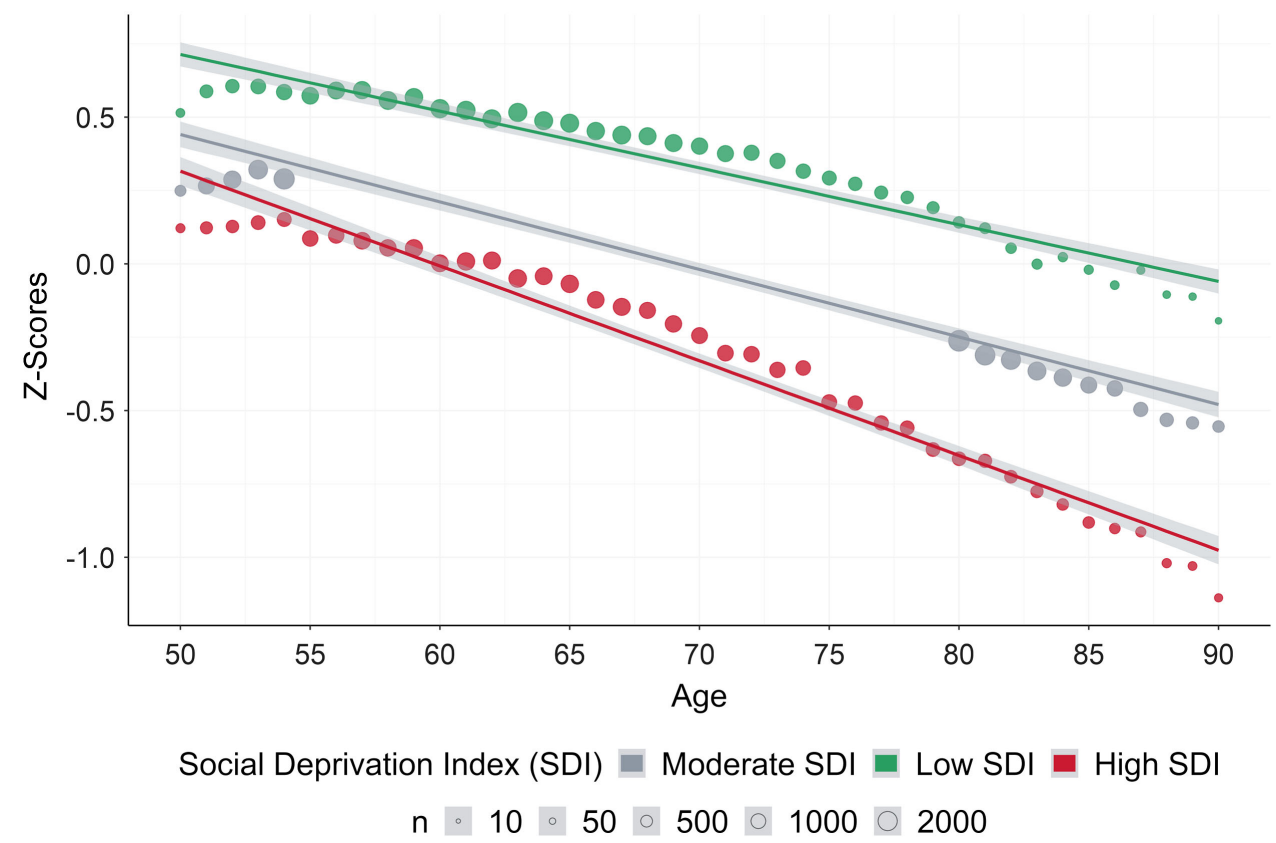

Figure 2. Average cognitive scores across ages 50-90 years by SoDep Index group. The regression lines illustrate the overall lower level of cognitive functioning in respondents categorized as experiencing high deprivation as compared to those moderate or low deprivation. Furthermore, they show the negative association between age and cognitive score. The size of the dots represents the number ( $\mathrm{n}$ ) of respondents whose scores counted toward the calculated average. Lines are the regression lines for respondents with high SoDep Index ( $80 \%$ percentile of SoDep Index scores; red) and with low SoDep Index (20\% percentile of SoDep Index scores; green). The gray color represents moderate SoDep Index scorers. Light gray shading surrounding regression lines shows the $95 \%$ confidence interval.

These associations survived adjustment for covariates. In Model 5, a unit increase in SoDep Index was associated with a cognitive score 0.037 SDs smaller $(\beta=-0.037, \mathrm{p}<.001)$ and a cognitive decline 0.003 SDs per year faster $(\beta=-0.003$, $\mathrm{p}<.001)$. Further, men had lower cognitive scores than women. Compared to being married or living with a partner, being widowed was associated with poorer cognition, being divorced with better cognition. The number of chronic conditions and the EURO-D score were negatively associated with cognitive score.

\section{Effect estimation}

We found that, at the age of 60 years, the predicted cognitive score for someone in the least deprived group (SoDep Index score at the lower 20\% mark) was 0.42 SDs above the average. At 80 years old, such an individual is expected to score 0.12 SDs above the average. Conversely, for someone in the most deprived group (SoDep Index score at the higher $20 \%$ mark), the predicted score was 0.09 SDs above the average at 60 years old and 0.29 SDs below the average at 80 years old. Figure 2 illustrates the cognitive decline in those extreme SoDep Index groups compared to respondents with moderate SoDep Index across the ages from 50 to 90 years.

\section{Sensitivity analysis}

Sensitivity analysis largely confirmed the results of the main analysis (see Table S3). The effect of SoDep Index $x_{\text {new }}$ was more pronounced than that of SoDep Index in the main analysis. A unit increase in SoDep Index new $_{\text {was }}$ associated with a cognitive score 0.241 SDs smaller $(\beta=-0.241, \mathrm{p}<.001)$ and a cognitive decline of 0.004 SDs per year faster $(\beta=-0.004, \mathrm{p}<.001)$. The negative associations between male gender, being widowed, and the EURO-D score with cognition remained comparable. So did the positive association between being divorced and cognition. However, the number of chronic conditions, which showed a negative association in the main analysis, had no association with cognition.

\section{Discussion}

In this paper, we aimed to validate the association between an index of social deprivation (SoDep Index), originally derived in the US American sample, and cognitive function and decline in a European sample (Hofbauer and Rodriguez, 2021). In an additional sensitivity analysis, we aimed to construct a more comprehensive index (SoDep Index new $_{\text {) with }}$ additional deprivation domains. Findings indicated 
that the original SoDep Index score (summarizing years of education, income, wealth, job stability, and health insurance status) indeed also predicts cognitive function and decline in the European sample. This effect was evident even when adjusting for a range of covariates relevant to cognitive function. Effect estimation showed that the effect on cognitive status is more pronounced than that on rate of decline. Results of our previous analysis were therefore replicated in this separate sample. Moreover, the sensitivity analysis showed that the SoDep In$\operatorname{dex}_{\text {new }}$ (summarizing income, wealth, inability to afford groceries, inability to afford a holiday, inability to afford an unexpected expense, inability to replace worn-out clothing, inability to replace worn-out shoes, and missing a necessary doctor appointment to save cost) had even greater explanatory power than the SoDep Index in the present sample. This underlines the importance of financial security to cognitive function and decline.

These results also are in line with previous observations that showed that indicators of socioeconomic status predict cognitive status (Chiao et al., 2014; Lövdén et al., 2020; Lyu and Burr, 2016; Marden et al., 2017) and with a previous investigation linking social deprivation and subjective cognitive decline (Bongue et al., 2016). The less pronounced association with cognitive decline echoes the mixed findings to date on the connection of indicators such as education with cognitive decline (Lövdén et al., 2020). This suggests that social deprivation mainly affects later adult cognitive functioning through a lowering of the cognitive peak that is achieved. The speed of decline then appears to be comparable to that found in less deprived individuals.

To understand how the link between SoDep Index and cognition can be explained, we consider the SoDep Index components. Education, for one, is robustly linked to enhanced brain structure and function (Arenaza-Urquijo et al., 2013) and, importantly, enhanced cognitive reserve resulting in better cognitive functioning even in the presence of neurodegeneration (Meng and D'arcy, 2012). Higher education also positively affects health behaviors, which will further promote good health (Feinstein et al., 2006). The association between health insurance status and cognitive function is also clear, as increased access to health care will contribute to improved health outcomes and thus an overall reduced cumulative health risk (Sommers et al., 2017). When health risk factors for cognitive decline are monitored and managed by health professionals, this can be assumed to be associated with improved cognitive status (Hussenoeder and Riedel-Heller, 2018).

The role of income and wealth in cognitive function is maybe less obvious. At the same time, these appear particularly robust indicator domains of social deprivation, showing loadings on both the

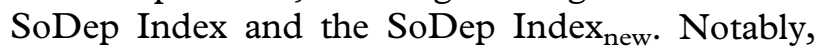
the SoDep Index $x_{\text {new }}$ suggests that most relevant aspects to deprivation are linked to financial security. One possible explanation is that financial security may affect cognitive function through the reduction of chronic stress. Chronic stress leads to systemic inflammation, harming the whole body, including the brain, which results in poorer cognition (Lupien et al., 2005). Financial security also determines the extent to which an individual will be able to afford a health-promoting lifestyle (Feinstein et al., 2006).

Our findings reinforce the importance of reducing social deprivation, which is a declared political aim of the European Union (European Commission, 2010). Comparing the incidence of high deprivation markers across countries shows that some countries are more notably affected, such as Greece and Poland. While improving living standards should remain a pan-European goal, these findings suggest that certain countries would benefit especially from support. Greece, in particular, has been affected by the financial crisis, and a direct link with poorer population health has been observed (Vandoros et al., 2013). In line with this, Greece is currently second only to Italy in terms of dementia prevalence (Alzheimer Europe, 2019), so that any intervention resulting in a reduction of dementia burden should be particularly relevant to national policy-makers.

In its current form, the SoDep Index ${ }_{\text {new }}$ has broad applicability across European countries, which could be useful for further pan-European research into social deprivation and comparative research across the globe. However, such investigations could be complemented by the work focused on the development of country-specific social deprivation indices. Such indices would be useful to capture the deprivation domains particularly relevant to each individual societal context. For instance, while job stability did not emerge as a

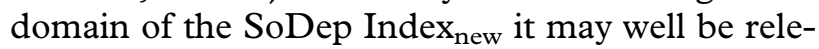
vant to the construction of a country-specific index for some central European countries with comparatively rigid job markets. In these countries, for example, the Netherlands and Spain, losing one's job is more likely to result in long-term unemployment (Hobijn and 凶ahin, 2009; Storm and Naastepad, 2009). Similarly, the SoDep Index ${ }_{\text {new }}$ does not include the inability to afford heating, but this item may show greater relevance in countries with high rates of so called "energy poverty." When adequate heating of the living space cannot be achieved, this is reflected in poorer physical and mental health of residents and excess mortality (Liddell and Morris, 
2010). Particularly high rates of energy poverty are observed in, for example, Bulgaria and Portugal (Bouzarovski, 2014).

As the SoDep Index ${ }_{\text {new }}$ comprises only eight domains, the questions underlying these domains could be administered in the form of a brief social deprivation scale in clinical settings. By assessing an individual's level of social deprivation, a clinician may be alerted to an increased risk of not only of cognitive decline but also of poorer general mental and physical health (Chung et al., 2018; Pförtner and Elgar, 2016; Saito et al., 2014). Future research may investigate the psychometric properties of such a screening tool. However, the usefulness of a social deprivation scale is restricted by the limited ability of any one individual to intervene in the domains of social deprivation. That is, deprivation in terms of the scale would have to be addressed at the societal level. As such, this research can be considered relevant to broader debates regarding socioeconomic security, for example, that surrounding the idea of an unconditional basic income. Future analyses could set out to estimate the economic cost of addressing social deprivation as compared to the cost of not addressing it.

Strengths of this study are the large, longitudinal population-based sample, and the availability of deprivation data that allowed for sensitivity analysis. Yet, this study is not without limitations. Considering only a selection of social deprivation domains, we cannot exclude the possibility that the observed association is mediated by other factors pertinent to cognitive function, such as innate ability. However, our findings still open up the possibility of rapidly assessing risk for low cognitive function without the assessment of innate ability. Further, cognitive tests tend to have a low sensitivity to subclinical intraindividual decline, for example, the word fluency task ( $\mathrm{Li}$ et al., 2017). However, this suggests that our findings may under- rather than overestimate an association between social deprivation and cognitive decline.

In sum, our analyses emphasize an important association between social deprivation and cognitive status and decline. That the association is stronger with cognitive status than with cognitive decline suggests that policy-makers might potentially achieve greater effects by intervening early and thus hopefully raising the cognitive peak achieved by individuals.

\section{Acknowledgments}

We thank the SHARE staff for making the data available for analysis.

\section{Conflict of interest}

None.

\section{Description of authors' roles}

LMH and FSR conceptualized the study and undertook the literature search. Both developed the data analysis plan and had access to the data, which was provided by the Survey of Health, Ageing, and Retirement in Europe (SHARE). Data analysis and figure creation were undertaken by $\mathrm{LMH}$ and reviewed by FSR. LMH and FSR have both contributed to the writing and editing of the final manuscript and have approved of its submission.

\section{Supplementary material}

For supplementary material accompanying this paper visit https://doi.org/10.1017/S10416102210 00995

\section{References}

Alzheimer Europe. (2019). Dementia in Europe Yearbook 2019: Estimating the Prevalence of Dementia in Europe. Alzheimer Europe.

Arenaza-Urquijo, E. M., et al. (2013). Relationships between years of education and gray matter volume, metabolism and functional connectivity in healthy elders. Neuroimage, 83, 450-457.

Becker, W., Norlén, H., Dijkstra, L. and Athanasoglou, S. (2020). Wrapping up the Europe 2020 strategy: a multidimensional indicator analysis. Environmental and Sustainability Indicators, 8, 100075.

Bennett, D. A., Schneider, J. A., Tang, Y., Arnold, S. E. and Wilson, R. S. (2006). The effect of social networks on the relation between Alzheimer's disease pathology and level of cognitive function in old people: a longitudinal cohort study. The Lancet Neurology, 5, 406-412.

Bloomberg, M., et al. (2021). Sex differences and the role of education in cognitive ageing: analysis of two UK-based prospective cohort studies. The Lancet Public Health, 6, e106-e115.

Bongue, B., Colvez, A., Amsallem, E., Gerbaud, L. and Sass, C. (2016). Assessment of health inequalities among older people using the EPICES score: a composite index of social deprivation. The fournal of Frailty \& Aging, 5, $168-173$.

Bouzarovski, S. (2014). Energy poverty in the European Union: landscapes of vulnerability. Wiley Interdisciplinary Reviews: Energy and Environment, 3, 276-289.

Bradshaw, J. and Finch, N. (2003). Overlaps in dimensions of poverty. Fournal of Social Policy, 32, 513-525.

Brandt, J., Spencer, M. and Folstein, M. (1988). The telephone interview for cognitive status. Neuropsychiatry, Neuropsychology, and Behavioral Neurology, 1, 111-117. 
Chanfreau, J. and Burchardt, T. (2008). Equivalence Scales: Rationales, Uses and Assumptions. Edinburgh: Scottish Government.

Cherbuin, N., Kim, S. and Anstey, K. J. (2015). Dementia risk estimates associated with measures of depression: a systematic review and meta-analysis. BMF Open, 5, e008853.

Chiao, C., Botticello, A. and Fuh, J.-L. (2014). Lifecourse socio-economic disadvantage and late-life cognitive functioning in Taiwan: results from a national cohort study. International Health, 6, 322-330.

Chung, R. Y.-N., et al. (2018). Deprivation is associated with worse physical and mental health beyond income poverty: a population-based household survey among Chinese adults. Quality of Life Research, 27, 2127-2135.

European Commission. (2010). Europe 2020: A Strategy for Smart, Sustainable and Inclusive Growth: Communication from the Commission. Brussels, Belgium: Publications Office of the European Union.

Feinstein, L., Sabates, R., Anderson, T. M., Sorhaindo, A. and Hammond, C. (2006). What are the effects of education on health. Measuring the effects of education on health and civic engagement: Proceedings of the Copenhagen symposium, 2006). Organisation for Economic Co-operation and Development Paris, 171-354.

Fiest, K. M., et al. 2016). The prevalence and incidence of dementia due to Alzheimer's disease: a systematic review and meta-analysis. Canadian fournal of Neurological Sciences/fournal Canadien des Sciences Neurologiques, 43, S51-S82.

Galobardes, B., Shaw, M., Lawlor, D. A., Lynch, J. W. and Smith, G. D. (2006). Indicators of socioeconomic position (part 1). Fournal of Epidemiology $\mathcal{G}$ Community Health, 60, 7-12.

Gao, S., Hendrie, H. C., Hall, K. S. and Hui, S. (1998). The relationships between age, sex, and the incidence of dementia and Alzheimer disease: a meta-analysis. Archives of General Psychiatry, 55, 809-815.

Garber, A. M. and Skinner, J. (2008). Is American health care uniquely inefficient? Fournal of Economic Perspectives, 22, 27-50.

Grundy, E. and Holt, G. (2001). The socioeconomic status of older adults: How should we measure it in studies of health inequalities? Fournal of Epidemiology $\mathcal{E}$ Community Health, 55, 895-904.

Hobijn, B. and $\bowtie$ ahin, A. (2009). Job-finding and separation rates in the OECD. Economics Letters, 104, 107-111.

Hofbauer, L. M. and Rodriguez, F. S. (2021). Association of social deprivation with cognitive status and decline in older adults. International fournal of Geriatric Psychiatry, 36, 1085-1094.

Hooper, D., Coughlan, J and Mullen, M (2007). Structural equation modelling: guidelines for determining model fit. Electronic Fournal of Business Research Methods, 6, 53-60.

Hussenoeder, F. S. and Riedel-Heller, S. G. (2018). Primary prevention of dementia: from modifiable risk factors to a public brain health agenda? Social Psychiatry and Psychiatric Epidemiology, 53, 1289-1301.

Li, F., Takechi, H., Kokuryu, A. and Takahashi, R. (2017). Longitudinal changes in performance on cognitive screening tests in patients with mild cognitive impairment and Alzheimer disease. Dementia and Geriatric Cognitive Disorders Extra, 7, 366-373.

Liddel1, C. and Morris, C. (2010). Fuel poverty and human health: a review of recent evidence. Energy Policy, 38, 2987-2997.

Lövdén, M., Fratiglioni, L., Glymour, M. M., Lindenberger, U. and Tucker-Drob, E. M. (2020). Education and cognitive functioning across the life span. Psychological Science in the Public Interest, 21, 6-41.

Lupien, S. J., et al. (2005). Stress hormones and human memory function across the lifespan. Psychoneuroendocrinology, 30, 225-242.

Lyu, J. and Burr, J. A. (2016). Socioeconomic status across the life course and cognitive function among older adults: An examination of the latency, pathways, and accumulation hypotheses. Fournal of Aging and Health, 28, 40-67.

Mackenbach, J. P., et al. (2008). Socioeconomic inequalities in health in 22 European countries. New England fournal of Medicine, 358, 2468-2481.

Marden, J. R., Tchetgen Tchetgen, E. J., Kawachi, I. and Glymour, M. M. (2017). Contribution of socioeconomic status at 3 life-course periods to late-life memory function and decline: early and late predictors of dementia risk. American fournal of Epidemiology, 186, 805-814.

Meng, X. and D'arcy, C. (2012). Education and dementia in the context of the cognitive reserve hypothesis: a systematic review with meta-analyses and qualitative analyses. PloS One, 7, e38268.

Mueller, R. and Hancock, G. (2008). Best practices in structural equation modeling. In: J. Osborne (Ed.), Best Practices in Quantitative Methods (pp. 488-508). Thousand Oaks, CA: SAGE Publications, Inc.

Nebel, R. A., et al. (2018). Understanding the impact of sex and gender in Alzheimer's disease: a call to action. Alzheimer's E Dementia, 14, 1171-1183.

O'Reilly, D. (2002). Standard indicators of deprivation: do they disadvantage older people? Age and Ageing, 31, 197-202.

Pförtner, T.-K. and Elgar, F. J. (2016). Widening inequalities in self-rated health by material deprivation? A trend analysis between 2001 and 2011 in Germany. Fournal of Epidemiology and Community Health, 70, 82-89.

Saito, M., et al. (2014). Relative deprivation, poverty, and subjective health: JAGES cross-sectional study. PLOS One, 9, e111169.

Sommerlad, A., Ruegger, J., Singh-Manoux, A., Lewis, G. and Livingston, G. (2018). Marriage and risk of dementia: systematic review and meta-analysis of observational studies. Fournal of Neurology, Neurosurgery $\mathbb{E}$ Psychiatry, 89, 231-238.

Sommers, B. D., Gawande, A. A. and Baicker, K. (2017). Health insurance coverage and health-what the recent evidence tells us. The New England Fournal of Medicine, 377, 586-593.

Storm, S. and Naastepad, C. (2009). Labor market regulation and productivity growth: evidence for twenty OECD countries (1984-2004). Industrial Relations: $A$ Fournal of Economy and Society, 48, 629-654. 
Stuck, S., et al. (2020). SHARE release guide 7.1.1.

Townsend, P., Phillimore, P. and Beattie, A. (1988).

Health and Deprivation: Inequality and the North. Kent:

Croom Helm.

Vandoros, S., Hessel, P., Leone, T. and Avendano, $M$.

(2013). Have health trends worsened in Greece as a result of the financial crisis? A quasi-experimental approach. The European Fournal of Public Health, 23, 727-731.

Ward, D. D., Wallace, L. M. and Rockwood, K. (2021). Cumulative health deficits, APOE genotype, and risk for later-life mild cognitive impairment and dementia. Fournal of Neurology, Neurosurgery $\mathcal{E}$ Psychiatry, 92, 136-142. 